\title{
Development of Multi Diagonal based OCDMA System for Free Space Optical Communication System
}

\section{Dushyant Singh Chauhan}

Delhi Technological Univeristy, Delhi

Gurjit kaur ( $\sim$ gurjeet_kaur@rediffmail.com )

Delhi Technological University https://orcid.org/0000-0002-8487-1211

\section{Dinesh Kumar}

Delhi Technological University

\section{Research Article}

Keywords: Free space optics (FSO),), Multi access interference (MAI), Optical code division multiple access (OCDMA), Multi diagonal (MD) code, Zero cross correlation (ZCC)

Posted Date: July 19th, 2021

DOl: https://doi.org/10.21203/rs.3.rs-565252/v1

License: (c) (1) This work is licensed under a Creative Commons Attribution 4.0 International License. Read Full License 


\title{
Development of Multi Diagonal based OCDMA system for free space optical communication system
}

\author{
Dushyant Singh Chauhan \\ Department of Electronics \& \\ Communication Engineering \\ Delhi Technological University \\ New Delhi, India \\ dsc.niet@gmail.com
}

\author{
Gurjit Kaur \\ Department of Electronics \& \\ Communication Engineering \\ Delhi Technological University \\ New Delhi, India \\ gurjitkaur@dtu.ac.in
}

\author{
Dinesh Kumar \\ Department of Electronics \& \\ Communication Engineering \\ Delhi Technological University \\ New Delhi, India \\ dineshkumar@dtu.ac.in
}

\begin{abstract}
The performance of FSO communication link is subject to numerous atmospheric factors in wireless communication like fog, rainfall, and haze which leads to deteriorate the performance of a system in term of BER and power at the receiver side. Due to numerous advantages of OCDMA over other access techniques, it allows various users to access a channel simultaneously without intervention with the other user. It has the ability to provide security, large number of users, privacy, reduce interference from multiple users and operate asynchronously. So, in this paper, Multi-Diagonal codes along with the fiber brags grating filters for Spectral amplitude coded OCDMA technique is developed for FSO system and performance analysis is performed in terms of BER and received power.
\end{abstract}

Keywords-Free space optics (FSO),), Multi access interference (MAI), Optical code division multiple access (OCDMA), Multi diagonal (MD) code, Zero cross correlation (ZCC).

\section{INTRODUCTION}

Optical communication provides the solution towards increasing spectrum requirement. With the help of different multiple access techniques, the problem of huge spectrum requirement can be solved. These techniques provide sharing and access of spectrum efficiently. Generally, three access techniques have been used in optical communication i.e Optical Time Division Multiple Access (OTDMA) [1], Wavelength Division Multiple Access (WDMA) [2], and Optical Code Divison Multiple access (OCDMA). In OCDMA, for every user a unique code is assigned in an optical communication network. The major advantages [3] of OCDMA are that it can work asynchronously, can support larger number of users, flexible to add users in the network, provides security against eavesdropping, no need for controlling the existing network, and it does not require any scheduling. So, OCDMA is considered as the most promising technology. The performance of OCDMA network degrades due to various noises such as dark current noise, thermal noise, and multiple access interference (MAI) generated from multiple users. To minimize the effect of MAI [4], different code sequence are assigned to various users. The code sequence designed using Spectral Amplitude Coding-OCDMA (SAC-OCDMA), provides better solution which helps in reducing the effect of MAI. Various codes have been developed for SAC-OCDMA. Amongst these codes optical orthogonal codes (OOCs) offers better cross and auto- correlation properties as compare to other codes.

FSO communication captured great attention due to its high-speed line of sight communication, and utilizes a light beam to transmit information through atmospheric medium instead of optical fiber. Since it is an unguided medium, so due to its numerous advantages, it can be utilized in various telecommunication applications. It is very challenging to communicate at those places where laying down of fiber is a difficult. So FSO appears to be the best alternative for communication. In wireless communication, FSO have numerous advantages like, license-free communication, high data rate. But weather conditions plays a very crucial role in FSO communication. Due to bad weather the performance of the optical link gets affected [5]. Line of sight communication is affected by Fog and Rainfall condition, due to the decrease in visibility. For supporting many users simultaneously in shared media, OCDMA has been recognized as one of the most important technologies, and in some cases transmission capacity of an optical fiber can be increased by assigning unique code. OCDMA systems provides good data rate for small distances like within a city.

In literature, different orthogonal codes are available for implementing OCDMA technique [6]. Such as in [7] diagonal double weight (DDW), in [8] modified double weight (MDW) code, in [9] modified quadratic congruence (MQC) codes and in [10] multi-diagonal (MD) codes are implemented. MD code provides zero cross correlation amongst various users placed in the optical network, which supresses the effect of MAI due to other users, and offers flexibility in selecting the code weight for simultaneous users.

In this work, the Fiber Bragg Grating (FBG) dispersion compensation module is used to optimize the existing MD coded FSO system to achieve a better performance in terms of data rate. Furthermore, we compared the system performance under different weather conditions and analyse the system behaviour. The paper is organised as follow: we present in section 2 different attenuations due to weather conditions. In section 3, we present the MD code for our system. Section 4 is dedicated to system design \& simulation setup. Finally results in section 5 followed by the conclusion. 


\section{SPECIFIC ATtENUATION OF RAIN \& FOG}

Since FSO is a line of sight communication, so the performance depends on various environmental factors like rainfall, fog and snow. BER and received signal power gets affected due to attenuation present in the FSO medium. Amongst various environmental factor, atmospheric attenuation plays a very important role. Generally, attenuation due to fog dominates in atmospheric medium due to particle size comparable with the FSO system wavelength used. Scattering, absorption, fading and reflection can hinder or alter the optical characteristics. Environmental conditions can be predicted with the help of visibility. In order to compute the optical parameters from atmospheric parameters, several models describe the relationship between attenuation and visibility range are given in [11]. Specific attenuation in $\mathrm{dB} / \mathrm{km}$ is used to characterize the medium, and it can be calculated using equation (1)

$$
\beta(\lambda)=\frac{1}{D} 10 \log \left(\frac{P_{E}}{P_{D}}\right)=\frac{1}{D} 10 \log \left(e^{\gamma(\lambda) \mathrm{D}}\right)
$$

where $D(\mathrm{~km})$ is the length of link, $P_{E}$ is the emitted optical power, $P_{D}$ the optical signal power at distance $\mathrm{D}$, and $\gamma(\mathrm{D})$ the atmospheric attenuation coefficient. The effect of attenuation due to atmosphere is as follows:

\section{A. Fog}

Mie scattering theory is used to study the attenuation due to fog. However, detailed information and complex computations and required. A different approach depends on visibility is helpful in predicting atmospheric attenuation constant values. These values can be computed by implementing common empirical model. So, using equation (2), attenuation values due to fog can be calculated.

$$
\beta_{\text {fog }}(\lambda)=\frac{3.91}{\operatorname{Vis}}\left(\frac{\lambda}{550}\right)^{-q}
$$

where $\lambda(\mathrm{nm})$ is the wavelength used, Vis $(\mathrm{km})$ stands for visibility distance, and $q$ is known as size distribution coefficient of scattering, which can be calculated using Kim model [12].

$$
q= \begin{cases}1.6 & \text { Vis }>50 \\ 1.3 & 6<\text { Vis }<50 \\ 0.16 \text { Vis }+0.34 & 1<\text { Vis }<6 \\ \text { Vis }-0.5 & 0.5<\text { Vis }<1 \\ 0 & \text { Vis }<0.5\end{cases}
$$

Table 1 summarizes different values of specific attenuation in $\mathrm{dB} / \mathrm{km}$ based on visibility range for various atmospheric weather conditions [13].

TABLE 1 VISIBILITY RANGE AND ATTENUATION FOR FOG

\begin{tabular}{clcc}
\hline S.No & Weather type & $\begin{array}{c}\text { Visibility Range } \\
\mathbf{V}(\mathbf{k m})\end{array}$ & $\begin{array}{c}\text { Attenuation } \\
\mathbf{d B} / \mathbf{k m}\end{array}$ \\
1 & Light Fog & $0.77-1$ & 18.3 \\
2 & Very Light Fog & $1-1.8$ & 13.8 \\
3 & Light Mist & $1.9-2.5$ & 6.6 \\
\hline
\end{tabular}

\section{B. Rain}

Due to small size rain droplets, a scattering is produced which is known as wavelength-independent scattering. Also, there is a linear relationship between specific attenuation and rate of rain. The attenuation due to rate of rainfall $\mathrm{R}(\mathrm{mm} / \mathrm{hr}$ ) is given by equation (4).

$$
\beta_{\text {rain }}=1.046 R^{0.67}
$$

The specific attenuation for FSO medium under rainfall condition can also be computed using empirical formula given by equation(5).

$$
\alpha_{\text {rain }}=\frac{2.8}{V i s}
$$

Where Vis is visibility range in $\mathrm{km}$, table 2 shows the value of specific attenuation under different rainfall condition [13]. 


\begin{tabular}{|c|c|c|c|c|}
\hline S.No & Weather type & $\begin{array}{c}\text { Rainfall Rate } R \\
(\mathrm{~mm} / \mathrm{hr})\end{array}$ & $\begin{array}{c}\text { Visibility Range } \\
\text { Vis }(\mathbf{k m})\end{array}$ & $\begin{array}{c}\text { Attenuation } \\
\mathrm{dB} / \mathrm{km}\end{array}$ \\
\hline 1 & Strong Rainfall & 25 & $1.9-2$ & 6.9 \\
\hline 2 & Average Rainfall & 12.5 & $2.8-4.0$ & 4.6 \\
\hline 3 & Light Rainfall & 2.5 & $5.9-10$ & 2 \\
\hline
\end{tabular}

\section{MD CODE}

To achieve good performance in terms of BER and received power, codes for OCDMA systems must be unipolar with constant weight and minimum cross-correlation to reduce the effect of MAI. Hence, in [14] OOCs is a code family which provides low cross-correlation values are discussed. The notation for this type of code is $\left(\mathrm{L}, \mathrm{W}, \mathrm{l}_{\mathrm{a}}, \mathrm{l}_{\mathrm{c}}\right)$, where $\mathrm{L}$ is the length of code sequence, $\mathrm{W}$ is the weight, $\mathrm{l}_{\mathrm{a}}$ and $\mathrm{l}_{\mathrm{c}}$ is the auto-correlation and cross-correlation between any two codes.

In OCDMA networks, in a same time slot multiple simultaneously transmission can be performed through the FSO channel. Codeword must have minimum correlation, so as to avoid the effect of interference to achieve good performance. MD code is implemented in our design due to numerous advantages [15] i.e. its zero cross-correlation removes MAI, it shows further flexibility to select weight parameters and number of rows of a matrix as compared with other codes such as MQC. Apart from this it is easy to design and can support many users with a high rate of data. The main advantage of the code is in a way that overlapping does not occur for spectral characteristic of users.

For four users $\mathrm{K}=4$, code weight $\mathrm{W}=2$ the code length $\mathrm{N}=8$, and the corresponding MD code is given by (15).

$$
M D=\left[\begin{array}{llllllll}
1 & 0 & 0 & 0 & 0 & 0 & 0 & 1 \\
0 & 1 & 0 & 0 & 0 & 0 & 1 & 0 \\
0 & 0 & 1 & 0 & 0 & 1 & 0 & 0 \\
0 & 0 & 0 & 1 & 1 & 0 & 0 & 0
\end{array}\right]_{4 \times 8}
$$

So the codeword is achieved in terms of wavelength for individual users as per the coding technique:

$$
\text { Codeword }=\left\{\begin{array}{lll}
\text { user } 1 & \Rightarrow \lambda_{1}, \lambda_{8} \\
\text { user } 2 & \Rightarrow \lambda_{2}, \lambda_{7} \\
\text { user } 3 & \Rightarrow \lambda_{3}, \lambda_{6} \\
\text { user } 4 & \Rightarrow \lambda_{4}, \lambda_{5}
\end{array}\right.
$$

\section{SYSTEM DESIGN \& Simulation SETUP}

Two continuous wave lasers are used at the user side with an input power of zero $\mathrm{dBm}$ for individual user as per the weight of the code, an ideal $2 \times 1$ multiplexer is used to combine two different wavelengths corresponding to individual user. A user defined bit stream is modulated using NRZ modulation format with the help of MZ modulator. Power combiner or ideal mux is used to combine all the user information, which is further transmitted through the FSO medium. The FSO parameters are specified in table 3 and at the receiver end, an ideal de-multiplexer, a pair of dispersion compensated FBG is used as a decoder along with the optical adder to combine two different wavelengths, an APD and a low pass Bessel filter is used to recover and reconstruct back in to electrical form. Analyser is further used to calculate the BER and gives the information regarding eye patterns. Fig 1 shows the completed block diagram of MD codes FSO system. The simulation is performed using OptiSystem15 for 4 users with code weight of 2. Received power is also calculated under FSO system using equation (7), where $P_{R}$ and $P_{T}$ are received and transmitted power, $d_{R}$, and $d_{T}$ is receiver and transmitter aperture diameter (m), $\alpha$ is atmospheric attenuation $(\mathrm{dB} / \mathrm{km}), \theta$ is beam divergence $(\mathrm{mrad})$, and $D$ is the range $(\mathrm{km})$, whereas no atmospheric fading is considered while calculating the optical power.

$$
P_{R}=P_{T} \frac{d_{R}^{2}}{\left(d_{T}+\theta D\right)^{2}} 10^{-\alpha \frac{D}{10}}
$$

TABle 3 Simulation Parameters

\begin{tabular}{lll}
\hline S.No & Parameter & Values \\
\hline
\end{tabular}




\begin{tabular}{llc}
\hline 1 & Wavelength & $1550 \mathrm{~nm}$ \\
2 & Power & $-10 \mathrm{dBm}$ \\
3 & Number of users & 4 \\
4 & Range & 0.3 to $2.5 \mathrm{~km}$ \\
5 & Transmitted apperture diameter & $10 \mathrm{~cm}$ \\
6 & Received aperture diameter & $25 \mathrm{~cm}$ \\
7 & Divergence & $2 \mathrm{mrad}$ \\
8 & Bit Rate & $1 \mathrm{Gbps}$ \\
\hline
\end{tabular}

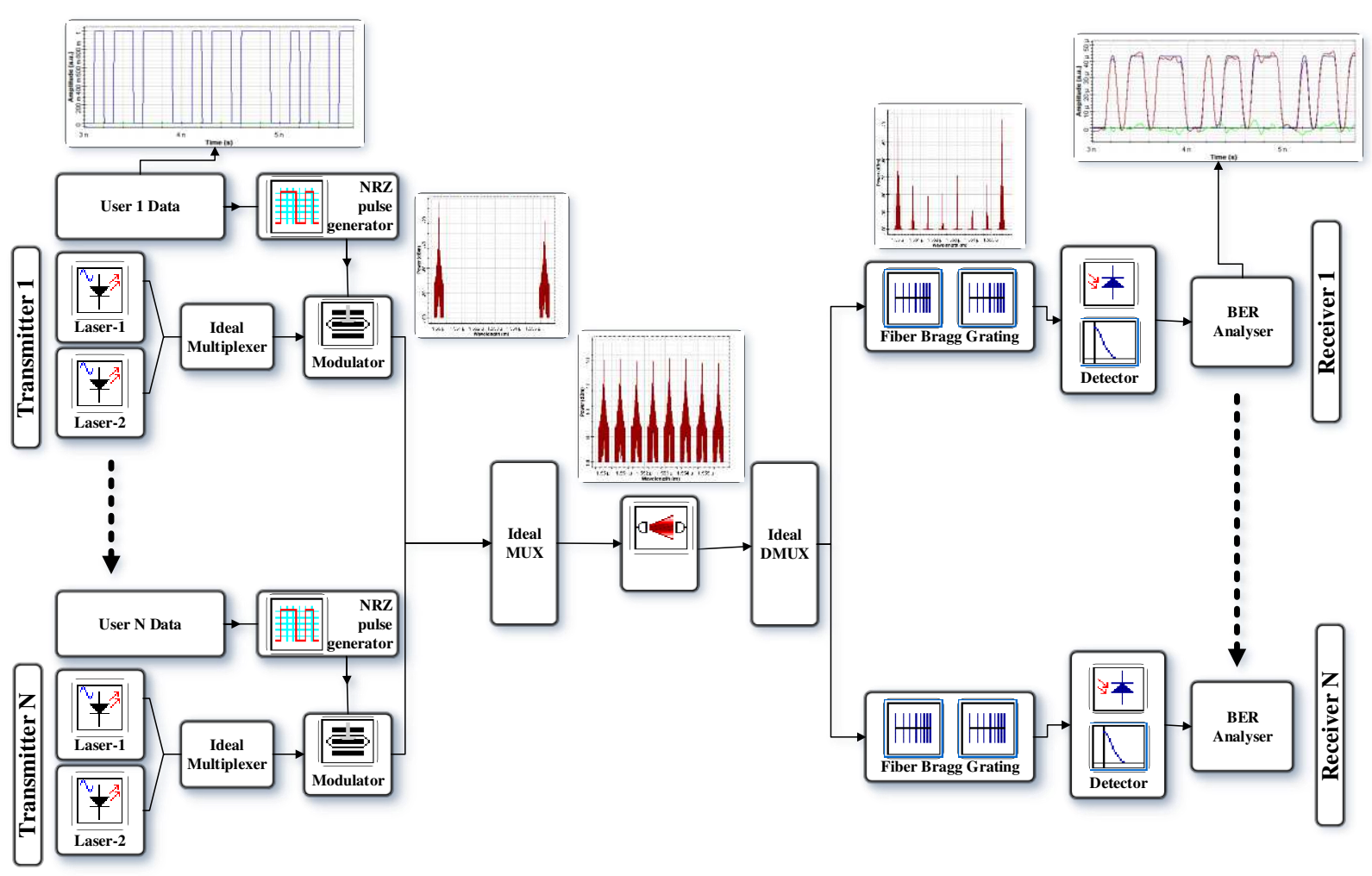

Fig. 1 Block diagram of MD coded FSO system

\section{RESULts}

A bit stream 0101101110 is transmitted through FSO and a similar bit pattern is observed on oscilloscope as shown in Fig.1. Transmitted distance is varied in FSO system and the design is investigated using MD code for different weather conditions. Fig.2 shows that with MD code the signals can transmitted upto $1.2 \mathrm{~km}$ with BER of $6.23 \times 10^{-11}$ under strong rainfall. Whereas a significantly high distance of $1.4 \mathrm{~km}$ with BER of $3.22 \times 10^{-13}$ is achieved under average rain condition. Under light rainfall, a distance further increased upto $2 \mathrm{~km}$ with BER of $7.23 \times 10^{-11}$. Fig.3 shows the variation of received power under different rainfall conditions for first user. 


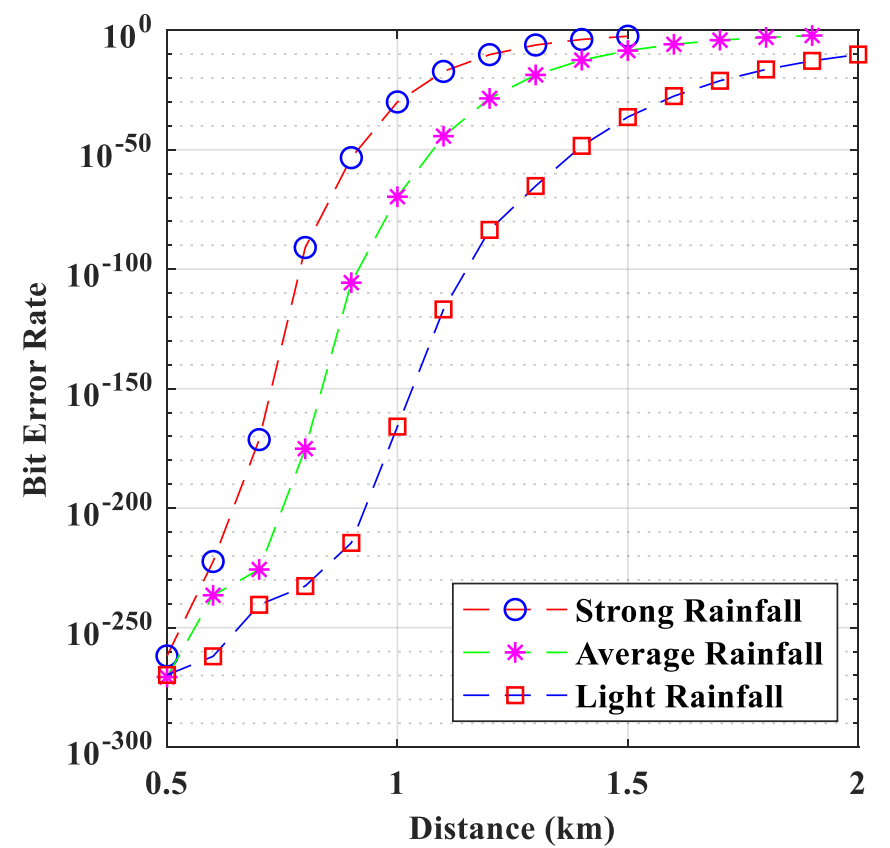

Fig. 2 BER vs range under different rainfall condition

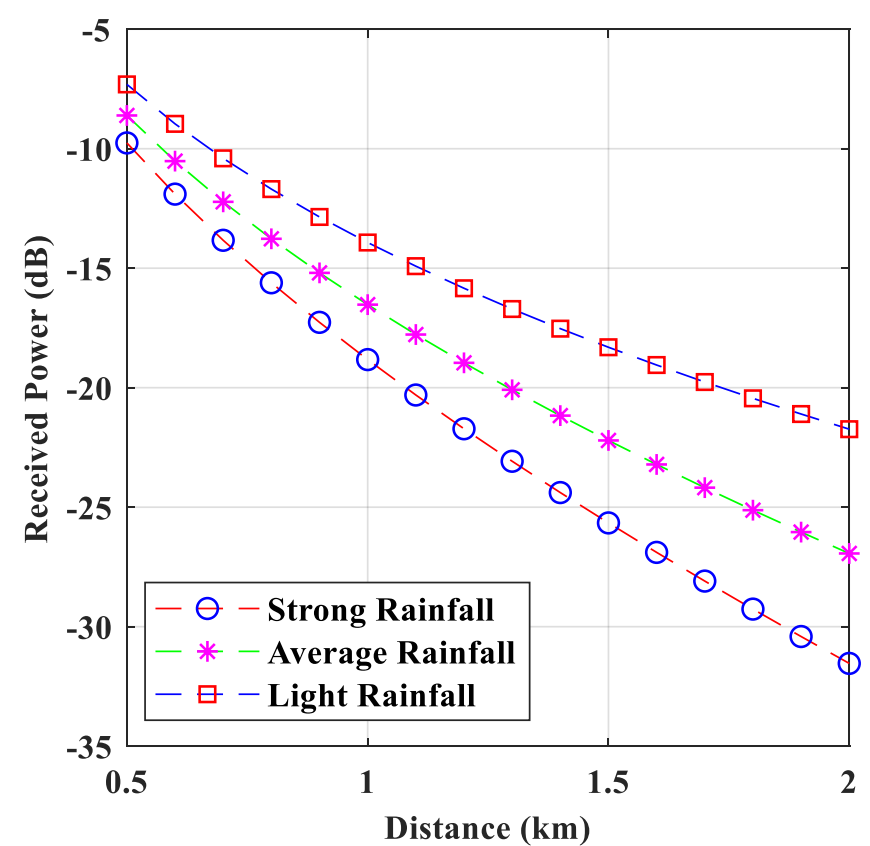

Fig. 3 Received Power vs range under different rainfall condition

The performance of MD coded OCDMA FSO network under different fog conditions is shown in fig 4, under light fog condition, a distance of $700 \mathrm{~m}$ is achieved with a significant BER value of $3.20 \times 10^{-13}$. In very light fog, distance of $800 \mathrm{~m}$ is achieved with BER of $2.09 \times 10^{-13}$. Fig.5 shows the variation of received power under different fog conditions. As distance increases received power decreases.

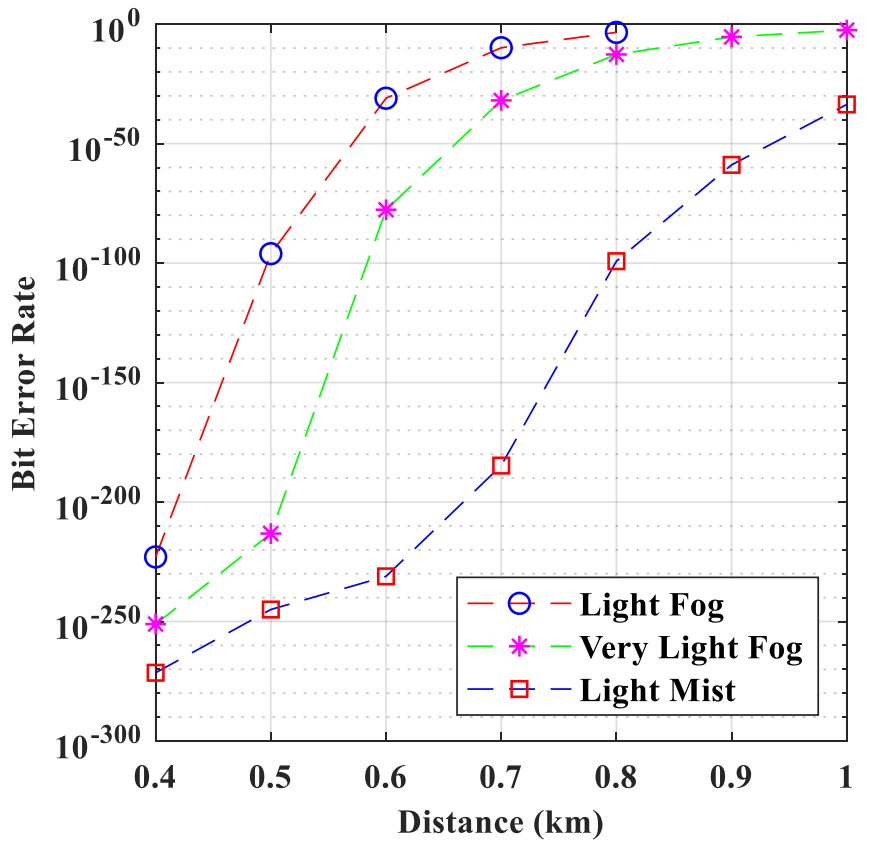

Fig. 4 BER vs distance under different fog condition

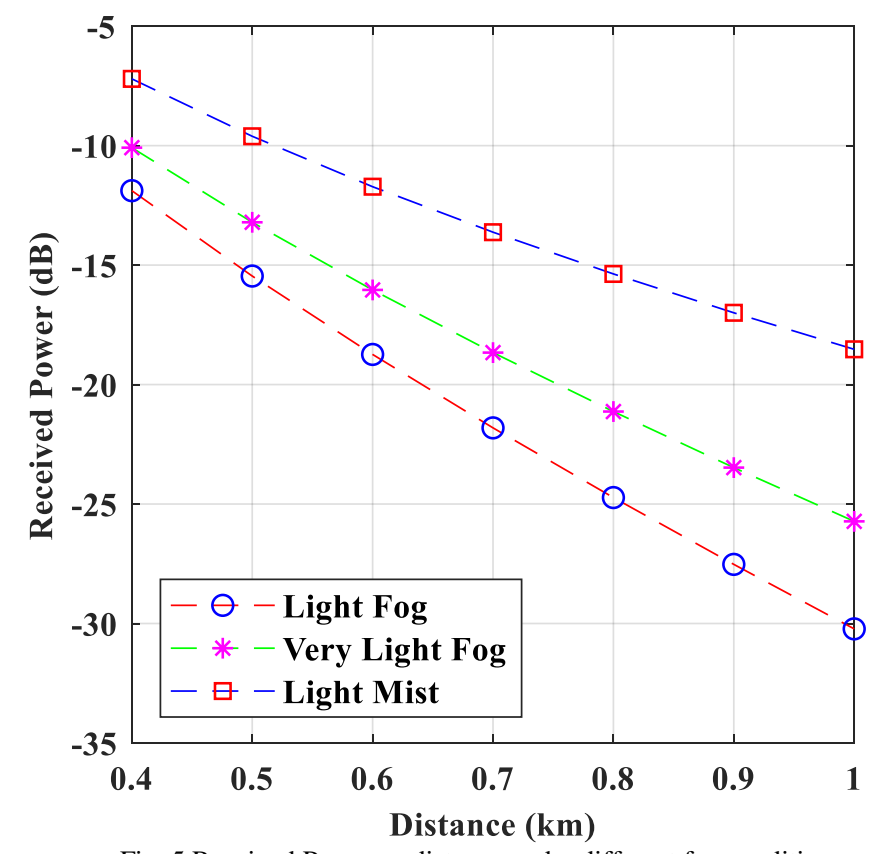

Fig. 5 Received Power vs distance under different fog condition

MD coded FSO system performs good upto a bit rate of $1.75 \mathrm{Gbps}$, fig. 6 shows variation of different bit rate. Under average rainfall condition a significant distance of $1.3 \mathrm{~km}$ is achieved with a significant BER of $2.46 \times 10^{-12}$. Beam divergence also affect the system performance, fig. 7 shows the performance of system under average rainfall condition. The eye diagram shows that at even for higher values of beam divergence, system performance is good with BER of $5.34 \times 10^{-12}$. 


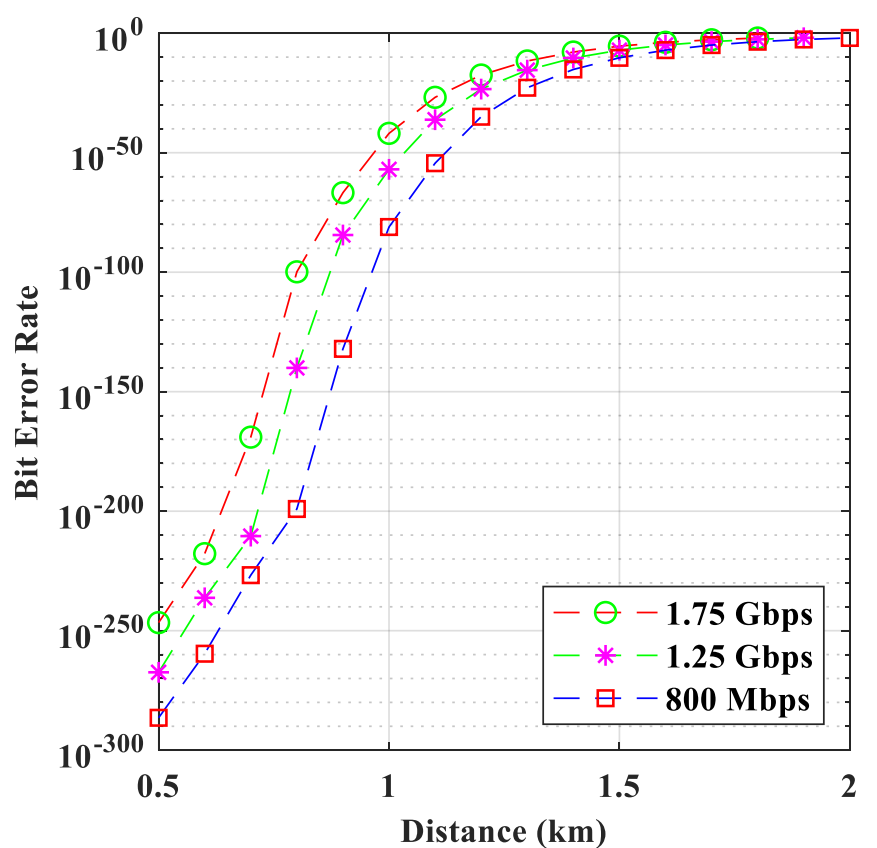

Fig. 6 BER vs distance at different bit rates under average rainfall condition

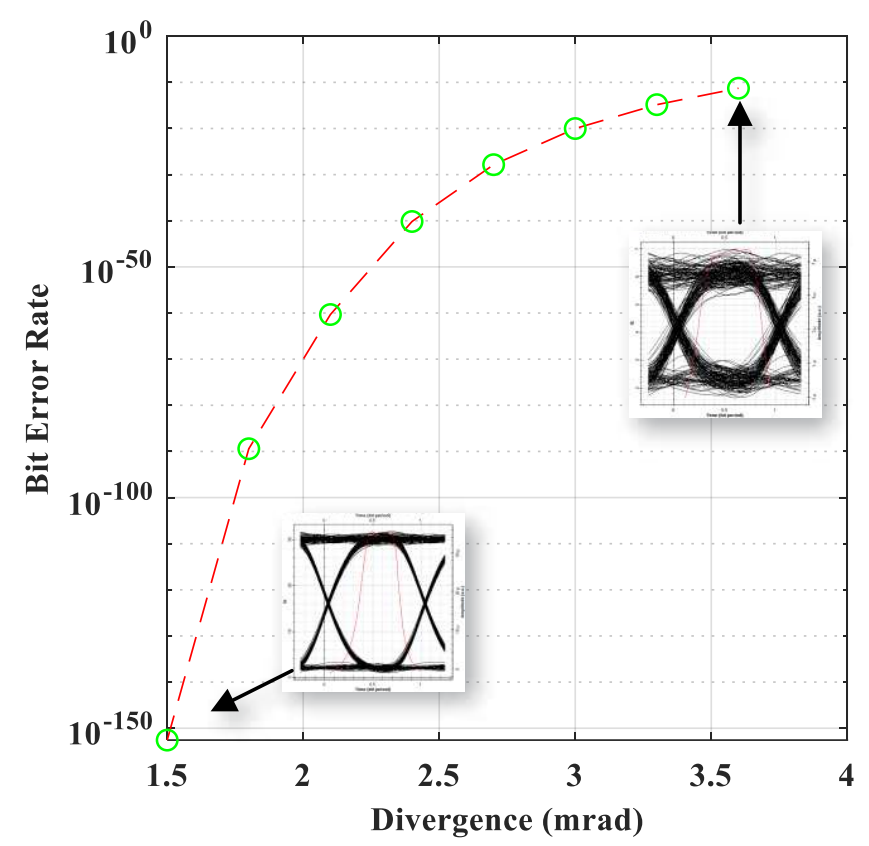

Fig. 7 BER vs beam divergence under average rainfall condition

\section{CONCLUSION}

The application of MD coded SAC-OCDMA technique in FSO system have numerous benefits such as security enhancement, flexible channel allocation and at any stage users can be increased. This paper discussed the development of MD OCDMA system \& its performance analysis for FSO medium. It illuminated the effect of rainfall and fog attenuation on the system performance along with the beam divergence. In addition, MD codes are ZCC codes, so it reduces the effect of MAI due to other users and outperforms the codes having low cross-correlation values. System performs well even at higher values of beam divergence. Distance achieved under strong rainfall and light fog conditions are $1.2 \mathrm{~km}$ and $700 \mathrm{~m}$.

\section{ACKNOWLWDGEMENT}

The authors would like to thank the "Research Project grant to the faculty of DTU" under Delhi Technological University for its financial support of this work under file No. DTU/Council/BOM-AC/Notification/31/2018/5738. The authors also would like to thank the anonymous reviewers for the feedback.

\section{REFERENCES}

[1] C.H. Yeh, B.S. Guo,Y.J. Chang,C.W. Chow,C.S. Gu, Bidirectional free space optical communication (FSO) in WDM access network with 1000-m supportable free space link. Optics Communications. (2019) Mar 15;435:394-8.

[2] M. Kumari, R. Sharma, A. Sheetal, Comparative Analysis of High Speed 20/20 Gbps OTDM-PON, WDM-PON and TWDM-PON for Long-Reach NG-PON2. Journal of Optical Communications. (2019).

[3] A.N. Islam, S.P. Majumder, Effect of Pointing Error on BER Performance of a Multi-wavelength OCDMA FSO System with SIK Dual Detector Receiver. Journal of Optical Communications. (2019) Jul 26;40(3):247-53.

[4] Y. Zouine, Z. Madini, Direct Sequence-Optical Code-Division Multiple Access (DS-OCDMA): Receiver Structures for Performance Improvement. InMultiplexing (2019) Sep 4. IntechOpen.

[5] P. Kundu,M.J. Islam,I.J. Talukder, Performance Analysis of Binary Polarization Shift Keying OCDMA FSO System under Different Atmospheric Conditions. In 2017 2nd International Conference on Electrical \& Electronic Engineering (ICEEE) (20170 Dec 27 (pp. 1-4). IEEE.

[6] X. Li, K.W. Shum, Mixed construction of OOC for optical code division multiple access networks. IET Communications. (2019) Apr 11;13(7):837-41.

[7] G. Kaur, Performance analysis of SAC-OCDMA in free space optical medium using MD and DDW code. In2015 2nd International Conference on Recent Advances in Engineering \& Computational Sciences (RAECS) (2015) Dec 21 (pp. 1-6). IEEE.

[8] M. Rani, H.S. Bhatti, V. Singh, Performance analysis of spectral amplitude coding optical code division multiple access system using modified double weight codes with Adomian decomposition method. Journal of Optical Communications. (2018) Oct 25;39(4):459-62.

[9] S. Mostafa, A.E. Mohamed, F.E. El-Samie, A.N. Rashed, Performance evaluation of SAC-OCDMA system in free space optics and optical fiber system based on different types of codes. Wireless Personal Communications. (2017) Sep 1;96(2):2843-61.

[10] R.A. Fayadh, M.K. Wali, M.F. Bonneya, Establishment Network by Using FSO Link Based on MD Code for Hybrid SCM-SAC-OCDMA Wireless System. International Journal of Electrical \& Computer Engineering (2088-8708). (2018) Dec 15;8.

[11] I. Kim, B. McArthur, E. Korevaar, Comparison of laser beam propagation at $785 \mathrm{~nm}$ and $1550 \mathrm{~nm}$ in fog and fog for optical wireless communications. Proc. SPIE 4214, 26-37 (2001)

[12] S.H. Alnajjar, A.A. Noori, A.A. Moosa, Enhancement of FSO communications links under complex environment. Photonic sensors. (2017) Jun 1;7(2):113-22.

[13] https://www.itu.int/dms_pubrec/itu-r/rec/../R-REC-P.1817-1-201202-I!!MSW-E.docx

[14] S. Kumawat, M.R. Kumar, A review on code families for sac-ocdma systems. InOptical and Wireless Technologies (2020) (pp. 307-315). Springer, Singapore.

[15] T.H. Abd, S.A. Aljunid, H.A. Fadhil, R.A. Ahmad, N.M. Saad, Development of a new code family based on SAC-OCDMA system with large cardinality for OCDMA network. Optical Fiber Technology. (2011) Jul 1;17(4):273-80. 